\title{
Influence of strings with axionic content on the polarization of extragalactic radio sources
}

\author{
Luis Masperi \\ Centro Atómico Bariloche and Instituto Balseiro \\ Comisión Nacional de Energía Atómica \\ 8400 Bariloche, Argentina \\ Sandra Savaglio \\ Dipartimento di Fisica, Università della Calabria \\ Arcavacata di Rende, 87036 Cosenza, Italy
}

\begin{abstract}
It is shown that the axion concentrated in electroweak strings which are consequently stabilized may produce a rotation of the polarization angle of radiation from extragalactic sources larger than that caused by a background field, and that axionic walls attached to global strings have an effect which depends on the frequency. We discuss the validity of the classical and quantum treatments of radiation and indicate that the latter produces conversion of linear into circular polarization. We describe possible anomalies in the observations which might suggest these influences of the axion.
\end{abstract}




\section{INTRODUCTION}

One of the last pieces which would complete the standard model of fundamental interactions is the axion, considered as the most elegant explanation for the absence of strong CP violation ${ }^{(1)}$. This particle would be a quasi-Goldstone boson caused by the spontaneous breaking of a global U(1) symmetry at an energy scale higher than the electroweak one. Its mass, due to instanton effects, is bounded in a window determined by astrophysical observa-

tions and cosmological arguments ${ }^{(2)}$, giving to the axion the possibility of being a candidate of CDM.

The interaction of the axion with the electromagnetic field affects differently right and left circularly polarized radiation so that the observation of extragalactic sources might give evidence for its existence. The consideration of background axion-like fields, corresponding however to extremely light quasi-Goldstone bosons, has allowed to establish bounds to their coupling with the electromagnetic field ${ }^{(3)}$.

In the present work we analyze the interaction of radiation with static condensates of axions either in the form of global strings, possibly stabilized by electroweak components, or of electroweak strings with axions in their core. For the latter case it is reasonable to think that the axions in the string core are very light both giving more stability to the string and making the treatment of the interaction with electromagnetic field more reliable. The coherent nature of axionic condensates in strings may produce a rotation of the polarization angle of radiation larger than for a background field.

In Section II we describe the classical interaction of radiation with static axionic field developing a perturbative treatment, which may be applied to rapid variations of the latter, and propose electroweak strings with axionic content. In Section III we indicate that the adiabatic quantum approximation reproduces the classical treatment and how the perturbative scattering of photons on a static axionic condensate may produce the conversion of linear to circular polarization. In Section IV we suggest possible astrophysical observations which might give evidence of the above effects. 


\section{CLASSICAL INTERACTION OF RADIATION WITH STRINGS}

The interaction of a very light pseudoscalar field $\sigma$ of the axion type with the electromagnetic field is

$$
L_{\mathrm{inf}}=-g \sigma \vec{E} \cdot \vec{B}
$$

For time-independent $\sigma$, small coupling constant $g$ and $\vec{\nabla} \sigma$ perpendicular to the electromagnetic fields $\vec{E}$ and $\vec{B}$, Eq. (1) gives way to modified Maxwell equations

$$
\begin{array}{r}
\square \vec{E}=-g \nabla_{i} \sigma \nabla_{i} \vec{B} \\
\square \vec{B}=g \nabla_{i} \sigma \nabla_{i} \vec{E}
\end{array}
$$

which, defining $\vec{F}_{ \pm}=\vec{E} \pm i \vec{B}$, are equivalent to

$$
\square \vec{F}_{ \pm}= \pm i g \nabla_{i} \sigma \nabla_{i} \vec{F}_{ \pm}
$$

If the variation of $\sigma$ is slower than that of the electromagnetic field allowing $\vec{\nabla} \sigma$ to be

taken as a constant, the two circularly polarized waves $\vec{F}_{ \pm}$travel with different velocities ${ }^{(4)}$, so that a linearly polarized wave traversing a width $L$ of the medium where the axion field changes in $\Delta \sigma$ will emerge with a polarization angle rotated in

$$
\Delta \theta=\frac{1}{2} g \Delta \sigma
$$

Considering $\sigma$ as a background field, and being the quasi-Goldstone boson of a symmetry broken at energy $f_{P Q}$, it has been estimated ${ }^{(3)}$ that the change of phase $\Delta \sigma / f_{P Q} \approx \pi$. In this way from the experimental uncertainty of $\theta$ it is possible to determine a bound for the coupling constant $g \simeq N \alpha / \pi f_{P Q}$, where $\alpha \simeq 1 / 137$ and $N$ integer.

The application of Eq. (4) seems more appropriate for static coherent configurations of solitonic type.

The simplest one is the global string where the field $\psi(x)=\exp \left(i \sigma(x) / f_{P Q}\right)$ behaves as a vortex for large distances from an axis for massless axion. If two incident linearly 
polarized electromagnetic rays pass perpendicularly to the string axis on each side of its core, according to Eq. (4) the variation of their polarization angle will be of opposite sign. If the global string may act as a gravitational lens due to its large mass the two images will have a difference in the polarization angle $\Delta \theta \approx g \pi f_{P Q}$ which is of a few degrees for small N.

On the other hand when the axion mass is taken into account the configuration requires walls attached to the string separating regions where $\sigma$ corresponds to different minima of the potential ${ }^{(5)}$. The walls attract one another finally leaving just one across which $\sigma / f_{P Q}$ changes in $2 \pi$. This variation of the axionic field is produced in the wall width $\varepsilon \simeq 1 / m_{a} \approx 1 \mathrm{~cm}$ for $m_{a} \simeq 10^{-5} \mathrm{eV}$. Therefore for radio frequencies the approximation which led to Eq. (4) is no longer valid and a different treatment is required.

For that purpose we build a perturbative method starting from Eq. (3). The zeroth order approximation is

$$
\square \vec{F}_{ \pm}^{(0)}=0 \quad, \quad \vec{F}_{ \pm}^{(0)}=\vec{f}_{ \pm}^{(0)} \exp \left[i\left(k_{0} z-\omega t\right)\right], \quad \omega=\left|k_{0}\right|
$$

such that if $f_{+}^{(0)}=f_{-}^{(0)}$ we have linear polarization.

If we consider the known case in which the variation of $\sigma$ is small in a wave-length, the first-order equation is

$$
\square \vec{F}_{ \pm}^{(1)}=\mp g \ell k_{0} \vec{F}_{ \pm}^{(0)} \quad, \quad \ell=\frac{\partial \sigma}{\partial z}
$$

whose Fourier transformation gives

$$
\vec{f}_{ \pm}^{(1)}(k)=\mp g \ell k_{0} \frac{\delta\left(k-k_{0}\right)}{k^{2}-\omega^{2}} \vec{f}_{ \pm}^{(0)}
$$

The difference of the variation of velocities for the right and left polarized waves defined by $\omega-k$ averaged with their Fourier component gives

$$
\frac{1}{2}\left[\int d k(\omega-k) \frac{f_{+}^{(1)}(k)}{f_{+}^{(0)}}-\int d k(\omega-k) \frac{f_{-}^{(1)}(k)}{f_{-}^{(0)}}\right]=\frac{1}{2} g \ell
$$

which multiplied times the path $L$ reproduces Eq. (4) checking the validity of the perturbative procedure. 
Passing to the opposite case where the change $\Delta \sigma$ is produced through a thin wall, narrow in comparison with the e.m. wave-length, the first-order equation is

$$
\square \vec{F}_{ \pm}^{(1)}=\mp g k_{0} \Delta \sigma \delta(z) \vec{F}_{ \pm}^{(0)}
$$

with the Fourier component

$$
\vec{f}_{ \pm}^{(1)}(k)=\mp \frac{g}{2 \pi} k_{0} \frac{\Delta \sigma}{k^{2}-\omega^{2}} \vec{f}_{ \pm}^{(0)} \quad, \quad \omega=k_{0}
$$

The physical effect of this perturbation comes from the average of $\omega-k$ as above of modes with positive $k$ for observations beyond the wall integrating up to the natural cut-off $\wedge=\frac{2 \pi}{\varepsilon}$ which produces a rotation of the polarization angle

$$
\Delta \theta=\frac{g}{m_{a} \lambda_{0}} \Delta \sigma \ln \left(1+m_{a} \lambda_{0}\right) \quad, \quad \lambda_{0}=2 \pi / k_{0}
$$

This shows a dependence of the effect on the wave-length at variance with the case of slowly varying $\sigma$.

A way to obtain larger effects on the polarization is to include the axion field in an electroweak string. This ${ }^{(6)}$ corresponds to a vortex of the Higgs field $\varphi$ compensated by the field $Z_{\mu}$ of the neutral vector boson. Though this configuration is metastable and even classically unstable $^{(7)}$ for realistic values of the Higgs mass, its stability can be increased by including the field $\sigma$ in a potential with two minima: the true vacuum corresponding to the phase with broken symmetry where $|\varphi|=v \sigma=0$ to avoid the strong $\mathrm{CP}$ violation and a false one for the symmetric phase existing inside the string where $\varphi=0 \sigma=\sigma_{0}$. This last non-vanishing value comes from the absence of one of the contributions which must be compensated by the axion field, i.e. $\arg \operatorname{det} M$ with $M$ mass matrix ${ }^{(8)}$. The local minimum decreases the string energy making its formation easier. On the other hand the fact that $\sigma / f_{P Q}$ is a phase allows excitations where its variation is $n 2 \pi$ along a closed string giving a quasi-topological reason for stabilization ${ }^{(9)}$. This is somehow similar to the superconducting cosmic strings ${ }^{(10)}$ but with the difference that there the excitations correspond to charged Goldstone-bosons coming from the breaking of $U(1)_{e m}$ in the core whereas in our case $\sigma$ 
inside the string may vary because the axions are lighter than outside, adjusting the potential parameters, for being related to a higher-temperature phase.

The string energy per unit length

$$
E=\int d^{2} x\left[(\vec{D} \varphi)^{*} \cdot \vec{D} \varphi+\frac{1}{2} \vec{\nabla} \sigma \cdot \vec{\nabla} \sigma+V(\varphi, \sigma)+\frac{1}{2}(\vec{\nabla} \times \vec{Z})^{2}\right]
$$

where $\vec{D}=\vec{\nabla}-i g^{\prime} \vec{Z}$ and a possible potential ${ }^{(9)}$ for $N=1$

$$
V=h\left(|\varphi|^{2}-v^{2}\right)^{2}+\left[m_{a}^{2} f_{P Q}^{2}+K\left(|\varphi|^{2}-v^{2}\right)\right]\left(1-\cos \frac{\sigma}{f_{P Q}}\right)
$$

may be estimated in thin-wall approximation

$$
E=a R^{2}+b R+\frac{c}{R^{2}}
$$

with $a$ determined by the difference of the two minima of potential, $b$ by the changes in the interface and $c$ by the Z-magnetic flux.

For the minimization of Eq. (14) with respect to the core radius $R$ one must fix the numerical values of the potential parameters. For a not too high Higgs mass it is reasonable to take $h=1$ together with the electroweak breaking scale $v=250 \mathrm{GeV}$. To give the expected axion mass in the broken symmetry phase $f_{p Q} \sim 10^{12} \mathrm{GeV} . \mathrm{K}$ is related to the axion mass inside the core

$$
m_{a}^{\prime 2}=K \frac{v^{2}}{f_{P Q}^{2}}-m_{a}^{2}
$$

One extreme position is i) to assume that when the core size is maximum the two minima of the potential are almost degenerate which occurs for $K=v^{2} / 2$. But in this way $m_{a}^{\prime} \simeq 10 \mathrm{eV}$ which does not seem sensible and requires larger energy to allow the oscillations of $\sigma$ along $z$. The opposite choice corresponds ii) to imagine that $m_{a}^{\prime}<m_{a}$ because the core has a higher symmetrical phase. In this case $K<10^{-8} \mathrm{GeV}$ and the oscillations of the core radius along $\mathrm{z}$ are very gentle. In any case due, to the fact that $\sigma$ oscillates inside the core, for an average situation we will take $b$ as depending only on the gradient of $\varphi$ in the interface whose width will have as upper bound $1 / m_{a}$. 
With the option i) the core radius may be as large as $0.1 p c$ with a linear energy density of $10^{31} \mathrm{gpc}^{-1}$ for a $Z$-magnetic field $B \simeq 10^{-1} G$. With the option ii) the second term of Eq. (14) becomes negligible compared with the first one and different almost constant core sizes are possible, e.g. $R \simeq 10^{-4} \mathrm{~cm}$, obviously with smaller $\varepsilon$, with $E \simeq 10^{20} \mathrm{gcm}^{-1}$ and an extremely high $Z$-magnetic field $B \simeq 10^{24} G$.

It is clear that the above numerical analysis is not necessarily realistic since it is based on the toy-model Eq. (13). Anyhow the general idea is that an electromagnetic wave which travels inside a large part of the string length, apart from the change of the polarization angle in the interface given by Eq. (11), will suffer an additional rotation according to Eq. (4) which might be larger than that produced by a background field, due to the coherent increase of $\sigma$ along the path.

\section{QUANTUM CONSIDERATIONS}

When the interaction Eq. (1) is taken as a source of quantum changes, one must distinguish between the adiabatic approximation according to which the photon adapts itself to the slow spatial changes that it encounters in the axionic medium, and the sudden transition to a different state.

In the first case one expects to obtain a result similar to the classical one which depends on the gradient of $\sigma$. Considering as interaction Hamiltonian

$$
H_{\mathrm{inf}} \simeq g \int d \vec{r} \sigma \vec{E} \cdot \vec{B}
$$

since the modification of the electromagnetic conjugate momentum is of order $g$, this interaction will change the quantum state of the photon as it moves through an axionic medium. Taking the photon as localized in a volume $\lambda^{3}$ over which the integration of Eq. (16) must be performed, at the beginning $\sigma=0$ to have linear polarization e.g. along $\vec{e}_{1}$. After the elementary path $\lambda=t$ the photon encounters a constant $\sigma$ slightly different from the initial one and, through the Schrödinger evolution operator, $H_{\text {int }}$ produces a component of polarization $\vec{e}_{2}$ in the quantum state with amplitude 


$$
\mathcal{A}=g \frac{\sigma}{2} \vec{e}_{1} \cdot\left(\vec{k} \times \vec{e}_{2}\right) t
$$

The rotation angle for the polarization of the photon may be defined as the expectation value of an operator $\vec{e}_{1} \times \vec{e}_{2}$ which will receive contribution only from the crossed term in the product of the final states, giving as result precisely Eq. (4). When the change of $\sigma$ appears only in a width $\varepsilon<\lambda$ the amplitude for the appearance of the polarization different from the initial one receives contribution for $t \sim \varepsilon$ so that, using Eq. (17) as a rough estimation, a rotation inversely proportional to $\lambda$ appears resembling Eq. (11).

If instead of considering electromagnetic waves travelling through an axionic medium we have the case of radiation polarized along $\vec{e}(\vec{k})$ impinging on a localized microscopic distribution of static $\sigma$, the perturbative treatment for sudden transitions to different states applies. Therefore, using Eq. (1), the cross-section for production of photons with polarization $\vec{e}^{\prime}\left(\vec{k}^{\prime}\right)$ is

$$
\frac{d \sigma}{d \Omega}=\frac{g^{2}}{16 \pi^{2}}\left(\vec{e}^{\prime}\left(\vec{k}^{\prime}\right) \cdot(\vec{k} \times \vec{e}(\vec{k}))\right)^{2}(\sigma(\vec{q}))^{2} k^{2}
$$

where $\sigma(\vec{q})=\int d \vec{r} \sigma(\vec{r}) e^{i \vec{q} \cdot \vec{r}} \quad, \quad \vec{q}=\vec{k}-\vec{k}^{\prime}$. If $\sigma \sim \sigma_{0}$ constant in the interaction volume $\mathcal{V}$, the maximum contribution of Eq. (18) will be for $\vec{k}=\vec{k}^{\prime}$ producing a possible conversion of linear to circular photon at the rate

$$
\left.\frac{d \sigma}{d \Omega}\right|_{\vec{q}=0}=\frac{g^{2}}{16 \pi^{2}} \sigma_{0}^{2} \mathcal{V}^{2} k^{4}
$$

which rises quickly with the frequency.

One may note that if $\sigma$ were a dynamical field the cross-section for photon scattering would result of order $g^{4}$ but this case is not realistic since the De Broglie wave-length of the axion is ${ }^{(2)}$ of order $10^{4} \mathrm{~cm}$.

Regarding the strings with axionic content, the simple global ones in terms of the field $\psi$ are finally attached to a domain wall and decay quantum mechanically ${ }^{(11)}$. But if they include electroweak components with two Higgs doublets their stability increases due to conservation of $Z$-magnetic flux ${ }^{(12)}$. Since the dynamics of axionic strings indicates ${ }^{(13)}$ that 
they would radiate mainly just before the QCD transition the earlier electroweak mechanism might stabilize a number of them. As for the electroweak strings which attract axions in their core according to Eq. (12-14), apart from the increase of classical stability given by the local potential minimum and the quasi-topological argument, complete quantum stability may be reached if axions are much lighter inside the core than outside it ${ }^{(14)}$. But this is possible for the original electroweak strings which survive till almost the QCD transition when the axion becomes massive.

\section{POSSIBLE RELATION WITH OBSERVATIONS}

Normally the polarization angle of radiation from extragalactic sources is at $0^{0}$ or $90^{0}$ with respect to the symmetry axis of the source and is rotated by Faraday effect giving a quadratic dependence on the wave-length for the observed angle(15)(16). However the statistical analysis of radiogalaxis with redshift $z>0.4$ shows $^{(15)}$ a possible excess in the distribution around $-40^{\circ}$ which, in case of being real, might be attributed to condensations of axionic field which affect some lines of sight.

Other observations which should be analyzed are those corresponding to close images whose sources are considered known so that their lines of sight are affected in a similar way by Faraday effect. This may happen for double-lobed sources which in some cases show unexplained polarization differences ${ }^{(17)}$. It would be analogously interesting to study the case of sufficiently close sources. As for the images of gravitational lenses, the difference of polarization angle is normally explained by Faraday effect ${ }^{(18)}$, being possible to speculate about the existence of examples where a global string acts as a lens.

In the emission of radiogalaxies there is a small percentage of circular polarization ${ }^{(17)}$ that in some abnormal cases increases with the frequency and which might be attributed to a conversion from linear to circular polarization by condensates of axionic matter.

There are also unexplained cases of polarization variations with time for constant total intensity ${ }^{(17)}$ which could be eventually due to the oscillation of electroweak strings with 
axionic content.

Finally in the optical region the dependence on the frequency is not clear and the Faraday effect is excluded ${ }^{(17)}$ so that there might be room for axionic effects which, as we have

seen, are independent on the frequency or proportional to it in case of rapid variation of axion field.

\section{Acknowledgments}

We are indebted to D. Harari and P. Veltri for interesting discussions. One of us (LM) thanks the Department of Physics of the University of Calabria at Cosenza for the hospitality during part of this work. This research has been partially supported by the grant PID 3965/92 of the Consejo Nacional de Investigaciones Científicas y Técnicas. 


\section{References}

1) R.Peccei and H.Quinn, Phys.Rev.Lett. $\underline{38}, 1440$ (1977);Phys.Rev.D $\underline{16}, 1791$ (1977).

2) E. Kolb and M. Turner, The Early Universe (Addison Wesley, Reading, MA, 1990).

3) D. Harari and P. Sikivie, Phys. Lett. B $\underline{289}, 67$ (1992).

4) J. Harvey and S. Naculich, Phys. Lett. B $\underline{217}, 231$ (1989).

5) P. Sikivie, Phys. Rev. Lett. $\underline{48}, 1156$ (1982).

6) T. Vachaspati, Nucl. Phys. B $\underline{397}, 648$ (1993).

7) M. James, L. Perivolaropoulos and T. Vachaspati, Phys. Rev. D $\underline{46}, \mathrm{R} 5232$ (1992).

8) G. Raffelt, Phys. Rep. 198, 1 (1990).

9) R. Fiore, D. Galeazzi, L. Masperi and A. Mégevand, Mod. Phys. Lett. A $\underline{9}, 557$ (1994).

10) E. Witten, Nucl. Phys. B $\underline{249}, 587$ (1985).

11) J. Preskill and A. Vilenkin, Phys. Rev. D $\underline{47}, 2324$ (1993).

12) G. Dvali and G. Senjanovič, Phys. Rev. Lett. $\underline{71}, 2376$ (1993).

13 R. Battye and E. Shellard, Axion string constraints, Cambridge preprint (1994).

14) L. Masperi and A. Mégevand, Stability of modified electroweak strings, Bariloche preprint (1994).

15) S. Carroll, G. Field and R. Jackiw, Phys. Rev. D $\underline{41}, 1231$ (1990).

16) A.Cimatti, S.Di Serego Alighieri, G.Field and R.Fosbury, Astrophys.J.422,562 (1994).

17) D.Saikia and C.Salter, Annu.Rev.Astron.Astrophys. $\underline{26}, 93$ (1988).

18) A. Patnaik, J. Browne, L. King, T. Muxlow, D. Walsh and P. Wilkinson, Mon.Not.R.Astron.Soc. 261, 435 (1993). 